\title{
EBV-BART-6-3p and cellular microRNA-197 compromise the immune defense of host cells in EBV-positive Burkitt lymphoma
}

\author{
YANG-MIN ZHANG ${ }^{1}$, YAN YU ${ }^{2}$ and HE-PING ZHAO ${ }^{2}$ \\ ${ }^{1}$ Department of Blood Transfusion, Xi'an Central Hospital, Xi'an, Shaanxi 710003; ${ }^{2}$ Clinical Laboratory of Hong-Hui Hospital, \\ Xi'an Jiaotong University College of Medicine, Xi'an, Shaanxi 710054, P.R. China
}

Received September 18, 2015; Accepted September 30, 2016

DOI: $10.3892 / \mathrm{mmr} .2017 .6173$

\begin{abstract}
The present study aimed to identify the association between Epstein-Barr virus (EBV) microRNA (miRNA) and cellular miRNA in compromising the immune system, which contributes to the development of Burkitt lymphoma (BL). The present study selected cellular miR-197 as the focus of the experiments due to the previous report that it is differentially expressed and the observation that interleukin-6 receptor (IL-6R) is a virtual target of miR-197 and EBV-BamHI A region rightward transcript (BART)-6-3p. In the present study, IL-6R was confirmed as a target of cellular miR-197 using a luciferase assay, and the negative regulatory association between miRNA (miR-197 and EBV-BART-6-3p) and mRNA (IL-6R) was confirmed by the observation that IL-6R was downregulated in EBV-positive Burkitt lymphoma and that miR-197 was upregulated. Additionally, mimics of EBV-BART-6-3p and miR-197 were introduced into lymphoma cells, and it was found that EBV-BART-6-3p and miR-197 synergistically reduced the expression of IL-6R. These findings improved current understanding of the role of miR-197/ EBV-BART-6-3p and their target, IL-6R, in the development of EBV-positive BL, and they may offer potential as novel therapeutic targets for the treatment of EBV-positive malignancies.
\end{abstract}

\section{Introduction}

Burkitt lymphoma (BL) is the first malignancy to be identified as being associated with viral infection, and viral infection demonstrates multiple steps in the development of malignancies $(1,2)$. Epstein-Barr virus (EBV) was first identified in the 1960s (3) and later, in the 1980s, EBV was found to be able

Correspondence to: Dr He-Ping Zhao, Clinical Laboratory of Hong-Hui Hospital, Xi'an Jiaotong University College of Medicine, 555 East Youyi Road, Xi'an, Shaanxi 710054, P.R. China

E-mail: hpzhaoebv@126.com

Key words: Epstein-Barr virus-BamHI A region rightward transcript-6, microRNA-197, interleukin-6 receptor, Epstein-Barr virus, Burkitt lymphoma to inserted into the human genome at the site of the $\mathrm{t}(9: 14)$ translocation, leading to c-MYC/Ig fusion and causing unlimited replication of the oncogene (4-6). In addition to c-MYC, certain other genetic lesions have been identified, which have been found to contribute to tumorigenesis $(6,7)$. Although the gene expression profile of BL is relatively homogeneous and different from that of other types of lymphoma, three classical variants of $\mathrm{BL}$ have been reported, including endemic BL, sporadic BL and immunodeficiency-associated BL (8), and the microRNA (miRNA) expression profile is relatively consistent in each type of BL (9).

miRNAs are a group of 22 nucleotide-long non-coding RNAs, which can be expressed by all eukaryotes and by several viruses (10). The transcription of miRNAs are generally catalyzed by RNA polymerase II to generate the precursor of the mature miRNA, termed the primary miRNA, which is sequentially processed by the RNase III enzyme, Drosha, in the nucleus to produce the pre-miRNA intermediate, and Dicer to yield the mature miRNA in the cytoplasm (11). miRNAs have been reported to be involved in the control of various biological processes, including proliferation, apoptosis, cell cycle and differentiation (12).

EBV encodes two clusters of miRNAs, which are differentially expressed in host cells with or without EBV infection (13). In host cells, which are positively infected with EBV, the virus generates relatively high levels of the three viral pre-miRNAs encoded in the miR-BamHI fragment $\mathrm{H}$ rightward open reading frame 1 (BHRF1) cluster and 22 pre-miRNAs encoded in the miR-BamHI A region rightward transcript (BART) cluster (14). In line with these findings, inactivation of the miR-BHRF1 cluster by mutagenesis markedly compromises B-cell transformation by EBV, and loss of all 22 miR-BARTs also has a significant impact on B-cell transformation (15-18). It has been reported that a member of the miR-BART cluster, EBV-BART-6-3p, targets interleukin- 6 receptor (IL-6R) in the host cells, and impairs the immune system, which is responsible for the transformation of B cells and lymphomagenesis (19). It has been found that, in addition to EBV-coded miRNAs, cellular miRNAs are also differentially expressed (19). In the present study, computational analysis was performed to identify the potentially expressed miRNAs, which may also target IL-6R, and their combined effects on the expression of IL-6R and the immune system were examined. 


\section{Materials and methods}

Sample collection. A total of $50 \mathrm{BL}$ samples, including 26 EBV-positive cases and 24 EBV-negative cases, were collected from Xi'an Central Hospital (Xi'an, China) between September 2013 and September 2014. The 50 BL samples consisted of 28 males (median age, 21) and 22 females (median age, 19) for the present study. The diagnosis of BL was established by at least two experienced pathologists. The present study was approved by the Ethics Committee of Xi'an Jiaotong University (Xi'an, China). Written informed consent was obtained from all participants.

Reverse transcription-quantitative polymerase chain reaction ( $R T-q P C R)$ analysis. MiRTarG (mirtar.mbc.nctu.edu.tw), miRanda (www.microrna.org), TargetScan (www.targetscan.org) were used in the present study. Tissues were homogenized using an Ultra-Turrax T-25 digital homogenizer (Daigger Scientific, Chicago, IL, USA). TRIzol reagent (Invitrogen; Thermo Fisher Scientific, Inc., Waltham, MA, USA) was used to isolate the total RNA, according to the manufacturer's protocol. An Oligo (dT) reverse transcription kit (Invitrogen; Thermo Fisher Scientific, Inc.) and $1 \mu \mathrm{g}$ total RNA were used to synthesize cDNA. The cDNAs were then amplified by qPCR using a SYBR Green qPCR kit (Takara Bio, Inc., Tokyo, Japan) on an Applied Biosystems 7500 Real-Time PCR system (Applied Biosystems; Thermo Fisher Scientific, Inc.). DNA polymerase was purchased from Takara Bio, Inc. and the sequences of th preimers used were: 5'-GGTCACGGG CACTCCTTGGAT AGGTACC-3' and 5'-CCCAGTGAGCTCCACCATCAAA-3'. miR-197, EBV-BART-6-3p and IL-6R mRNA were examined. The cycle protocol was as follows: $5 \mathrm{~min}$ at $95^{\circ} \mathrm{C}, 40$ cycles of $30 \mathrm{sec}$ at $95^{\circ} \mathrm{C}, 30 \mathrm{sec}$ at $58^{\circ} \mathrm{C}$ and $60 \mathrm{sec}$ at $72^{\circ} \mathrm{C}$. Dissociation curves were determined to evaluate the specific amplification of the PCR product. The $2^{-\Delta \Delta \mathrm{Cq}}$ method (20) was used to calculate the relative gene expression, with $G A P D H$ as an internal standard.

Western blot analysis. The lysates of the cultured cells or tissue samples were prepared using cell lysis reagent comprising $0.1 \%$ SDS, $1 \%$ Triton X-100, $130 \mathrm{mM} \mathrm{NaCl}$ and $50 \mathrm{nM}$ Tris- $\mathrm{HCl}(\mathrm{pH}$ 8.0) containing protease inhibitor cocktail (Sigma-Aldrich; Merck Millipore, Darmstadt, Germany) according to the manufacturer's protocol. Proteins were quantified using a BCA protein assay kit (Boster Biological Technology, Ltd., Wuhan, China). The proteins (30 $\mu \mathrm{g} / \mathrm{lane})$ were separated by $12 \%$ SDS-PAGE and transferred onto a nitrocellulose membrane (GE Healthcare Life Sciences, Little Chalfont, UK). The membranes were blocked with 5\% dried milk for $60 \mathrm{~min}$ to avoid unspecific binding. The signals on the blots were then detected by incubation with anti-IL6R (cat. no. sc-374259; 1:5,000; Santa Cruz Biotechnology, Inc., Dallas, TX, USA) and anti- $\beta$-actin antibodies (cat. no. sc-47778; 1:10,000; Santa Cruz Biotechnology, Inc.) at $4^{\circ} \mathrm{C}$ for $12 \mathrm{~h}$. Secondary antibody conjugated to horseradish peroxidase (cat. no. sc-516086; 1:10,000; Santa Cruz Biotechnology, Inc.) was used for incubation at room temperature for $2 \mathrm{~h}$ with the blots, following which an ECL Western Blotting kit (Applygen Technologies, Inc., Beijing,
China) was used for detection, according to the manufacturer's protocol. The relative density of the target bands on the blots was measured using desitometric analysis.

Cell culture. The ramos cell line was purchased from the Shanghai Cell Bank of the Chinese Academy of Sciences (Shanghai, China), and used for in vitro experiments. The cells were cultured in RPMI 1640 medium supplemented with $100 \mu \mathrm{g} / \mathrm{ml}$ streptomycin, $100 \mathrm{U} / \mathrm{ml}$ penicillin, $2 \mathrm{mM}$ glutamine and $10 \%$ fetal bovine serum (FBS; Lonza, Walkersville, MD, USA) at $37^{\circ} \mathrm{C}$ with $5 \% \mathrm{CO}_{2}$.

Oligonucleotide transfection. The ramos cells were incubated in a 6-well plate (Greiner Diagnostic GmbH, Bahlingen, Germany) at $1 \times 10^{6}$ cells/well in $2 \mathrm{ml}$ RPMI. Mimics of the cellular miR-197 and EBV-BART-6-3p were purchased from Shanghai GenePharma Co., Ltd. (Shanghai, China). When the cells grew to $70-80 \%$ confluence, the mimics or scramble controls were transfected into the ramos cells with transfectamin 2000 (Invitrogen; Thermo Fisher Scientific, Inc.), according to the manufacturer's protocol. Briefly, the PTENCE cells seeded in 48-well plates at a concentration of $1 \times 10^{5}$ cells/well for $12 \mathrm{~h}$, then Lipofectamine 2000 (Invitrogen; Thermo Fisher Scientific, Inc.) was used to transfect the cells with mimics of the cellular miR-197 and EBV BART-6-3p at $37^{\circ} \mathrm{C}$ for 4 to $6 \mathrm{~h}$.

Luciferase reporter assay. The full-length 3'untranslated region ( 3 'UTR) of IL-6R was amplified and inserted into multiple restrictive sites between the poly (A) site of the psiCHECK-2 plasmid (Promega Corporation, Madison, WI, USA) and the firefly luciferase-coding sequence. The amplification was carried out at $98^{\circ} \mathrm{C}$ for $60 \mathrm{sec}$, and followed by 30 cycles $\left(98^{\circ} \mathrm{C}\right.$ for $30 \mathrm{sec}, 58^{\circ} \mathrm{C}$ for $30 \mathrm{sec}, 72^{\circ} \mathrm{C}$ for $\left.120 \mathrm{sec}\right)$, and $72^{\circ} \mathrm{C}$ for $5 \mathrm{~min}$ on T100 PCR (Bio-Rad Laboratories, Inc., Hercules, CA, USA). A Quick Change mutagenesis kit (Stratagene, Heidelberg, Germany) was used to introduce mutations into the seed sequences of IL-6R. The ramos cells were seeded into a 96-well plate $(5,000$ cells/well) to evaluate the effects of miR-197 and miR-BART-6-3p on the expression of luciferase. After $24 \mathrm{~h}$, the cells were co-transfected with a psiCHECK reporter vector containing IL-6R and oligo miR-197, EBV-BART-6-3p or scramble controls. At $48 \mathrm{~h}$ post-transfection, the cells were assayed for luciferase activity using a Dual-Glo luciferase reporter assay system (Promega Corporation). Renilla luciferase activity was used to normalize firefly luciferase activity for each experiment.

Statistical analysis. Student's $t$-test or one-way analysis of variance were used to analyze data. Statistical analyses were performed using SPSS version 13.0 (SPSS, Inc., Chicago, IL, USA). $\mathrm{P}<0.05$ was considered to indicate a statistically significant difference. All results are expressed as the mean \pm standard deviation.

\section{Results}

$I L-6 R$ is a target of miR-197 and EBV-BART-6-3p. As a member of the IL family, the present study hypothesized that the IL-6R protein is involved in the EBV infection process. 
A

\section{Cellular miRNA}

IL-6R 3’ UTR (2838-2845)

Hsa-miR-197-3p

Mut IL-6R 3' UTR (2838-2845)

B

\section{EBV miRNA}

IL-6R 3’ UTR (797-803)

EBV-miR-BART6-3p

Mut IL-6R 3' UTR (797-803)

\author{
5' -TCTATGTTCTCTAATCTGTGGTGACT-3' \\ | | | | | | \\ 3' - CGACCCACCUCUUCCACCACUU - 5 ' \\ 5' -TCTATGTTCTCTAATCTCACCACTCT-3'
}

\author{
5' -GCACAGGGTCTCTACCATCCCCTGT-3' \\ 3' - AGAUUCCGAUCAGGCUAGGGGC-5' \\ 5' -GCACAGGGTCTCTACCTAGGGGTGT-3'
}

Figure 1. IL-6R is a target of miR-197 and EBV-BART-6-3p. (A) miR-197 binds to IL-6R on its putative binding site on the 3'UTR. (B) EBV miRNA miR-BART6-3p binds to IL-6R gene on a different site on the 3'UTR. miRNA, microRNA; IL-6R, interleukin-6 receptor; EBV, Epstein-Barr virus; Mut, mutant; 3'UTR, 3'untranslated region.
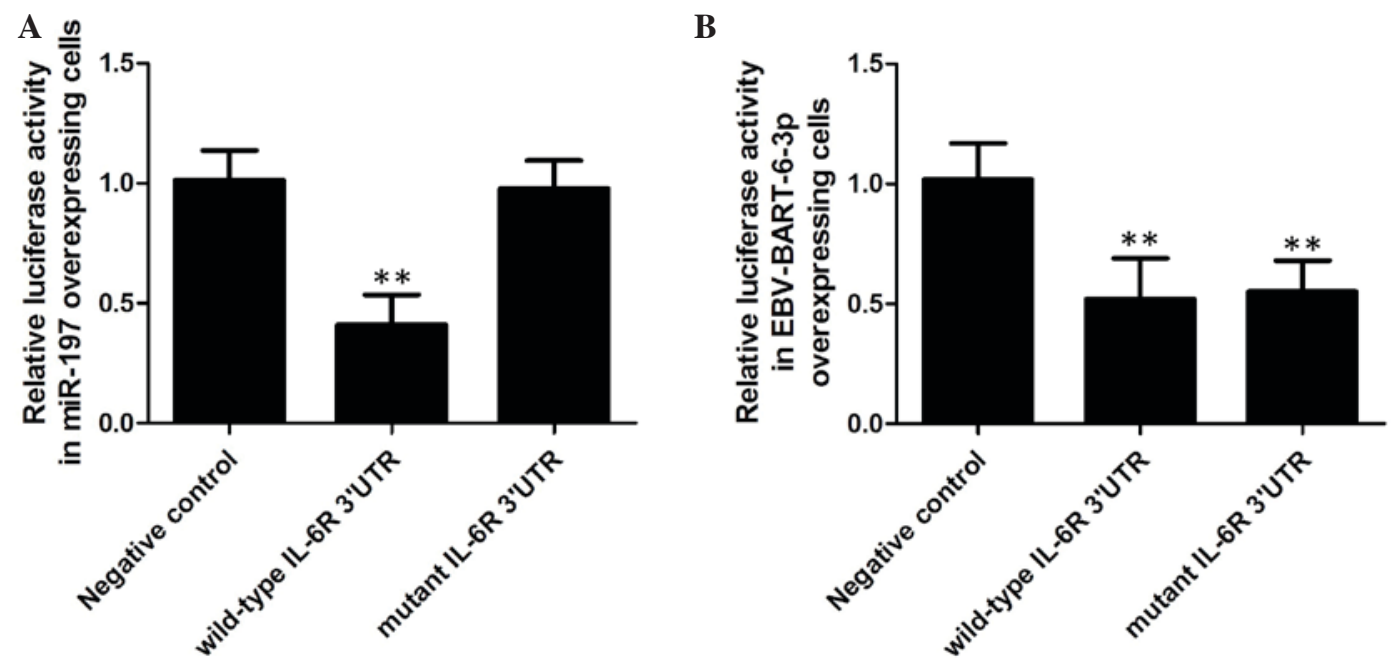

Figure 2. Effects of IL-6R on miR-197. (A) Relative luciferase activity of miR-197-overexpressing cells transfected with the wild-type IL-6R 3'UTR was lower, compared with cells transfected with mutant IL-6R 3'UTR and negative control. (B) Relative luciferase activities of miR-BART6-3p-overexpressing cells transfected with constructs containing wild-type/mutant IL-6R 3'UTRs were lower, compared with the negative control. ** P<0.01 vs. control group. miRNA, microRNA; IL-6R, interleukin-6 receptor; EBV-BART-6-3p, Epstein-Barr virus-BamHI A region rightward transcript-6-3p; Mut, mutant; 3'UTR, 3'untranslated region.

By searching an online miRNA database, IL-6R was identified as a target of miR-197 and EBV-BART-6-3p with putative binding sites in the 3'UTR of IL-6R (miR-197 binding site, 2,838-2,845 bp; EBV-BART-6-3p binding site, 797-803 bp). As shown in Fig. 1A and B, miR-197 matched the 3'UTR IL-6R at 2,838-2,845 bp, whereas EBV-BART6-3p matched the 3'UTR of IL-6R at 797-803 bp.

Overexpression of cellular/EBV miRNA inhibits the expression of $I L-6 R$. To confirm the association between the cellular/EBV miRNA and IL-6R, the present study performed a luciferase reporter assay with constructs containing wild-type and mutant IL-6R 3'UTR segments among groups of cells overexpressing miR-197 and EBV-BART-6-3p, respectively. As shown in Fig. 2A, the relative luciferase activity of the wild-type IL-6R 3'UTR was significantly downregulated, compared with the mutant IL-6R 3'UTR and negative control in the miR-197-overexpressing cells. In the cells overexpressing EBV-BART-6-3p, the wild-type and mutant IL-6R 3'UTR groups showed lower expression levels, compared with the negative control (Fig. 2B). The above findings indicated that cellular miRNA-197 downregulated the expression of IL-6R via targeting the mRNA putative binding sites $(2,838-2,845 \mathrm{bp})$.

miR-197 and EBV-BART-6-3p inhibit the expression of IL-6R in $E B V$-positive cells. To verify the regulatory association between EBV-coded miRNA/cellular miRNA and IL-6R, 


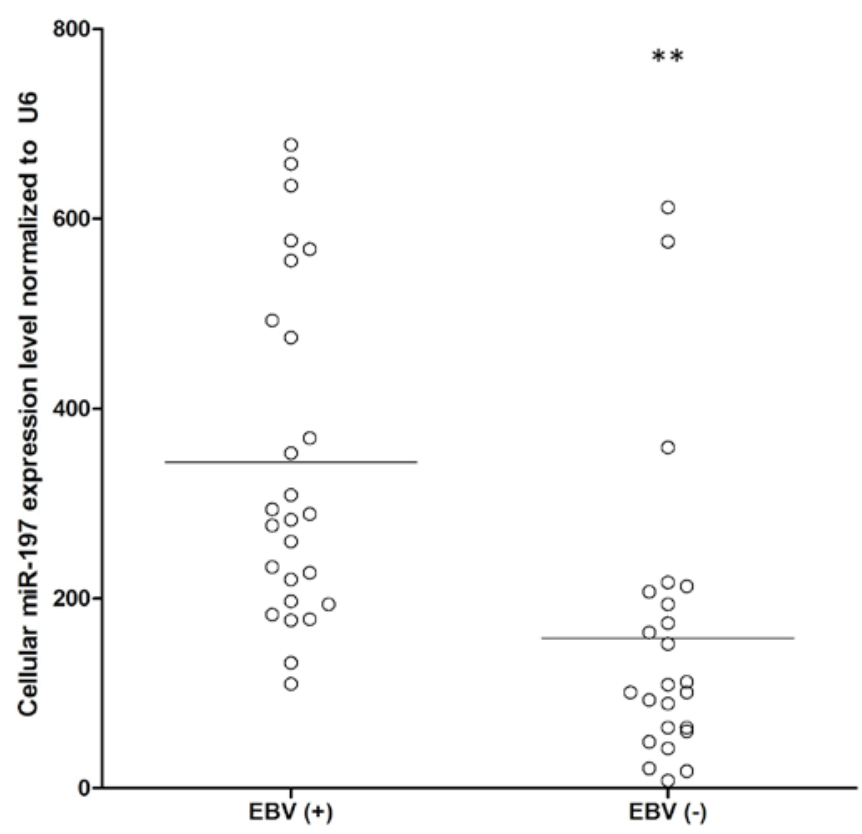

Figure 3. Expression of miR-197 in the presence and absence of EBV. Relative expression level of miR-197 was substantially higher in the EBV-positive tissue samples, compared with the EBV-negative samples.* $\mathrm{P}<0.01$ vs. control group. miR, microRNA; EBV, Epstein-Barr virus.
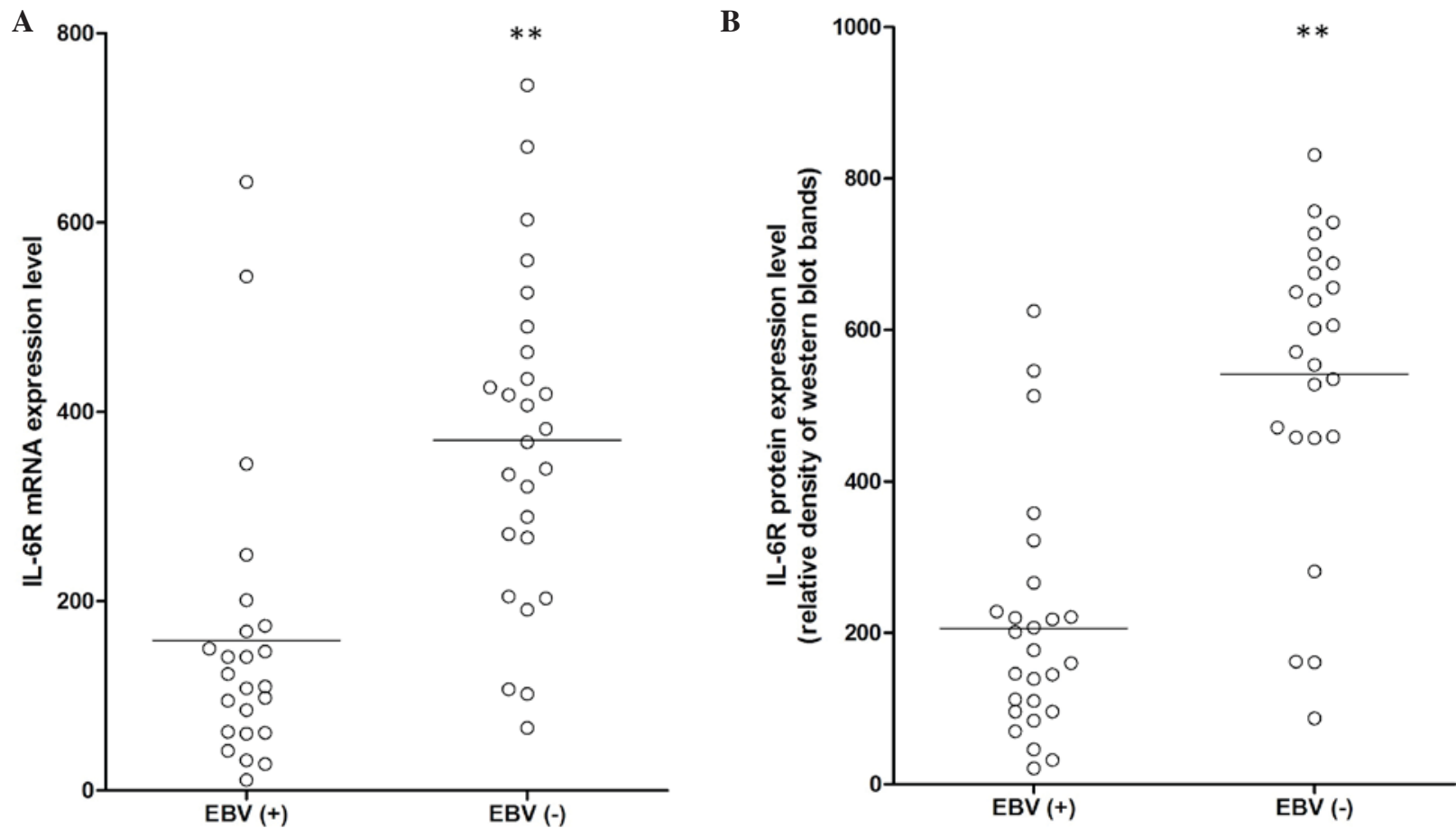

Figure 4. mRNA and protein levels of IL-6R in the presence and absence of EBV. (A) Relative mRNA expression level of IL-6R was markedly lower in the EBV-positive tissue samples, compared with the EBV-negative samples. (B) Western blot analysis revealed the relative density of IL-6R was decreased in the EBV-positive cell samples, compared with the EBV-negative cell samples. ${ }^{* *} \mathrm{P}<0.01$ vs. control group. EBV, Epstein-Barr virus; IL-6R, interleukin-6 receptor.

the present study examined the expression of miR-197 and IL-6R using RT-qPCR and western blot analyses. As shown in Fig. 3, the expression of miR-197 was markedly upregulated in the EBV-positive BL samples. Consistent with the differential expression of miR-197, the mRNA expression of IL-6R in the EBV-positive samples was lower, compared with the EBV-negative BL samples (Fig. 4A). The protein expression of IL-6R, as determined by the relative density of the western blot target bands, was decreased in the EBV-positive samples, compared with the EBV-negative samples (Fig.4B), indicating a possible negative regulatory association between IL-6R and miR-197/EBV-BART-6-3p.

Synergistic association between EBV-BART-6-3p and $m i R-197$. To verify the association between cellular miRNAs and EBV miRNA and their target, IL-6R, the present study 
A
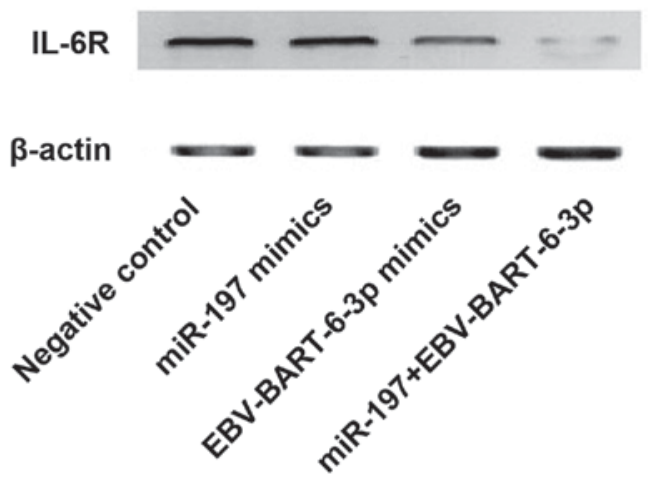

B

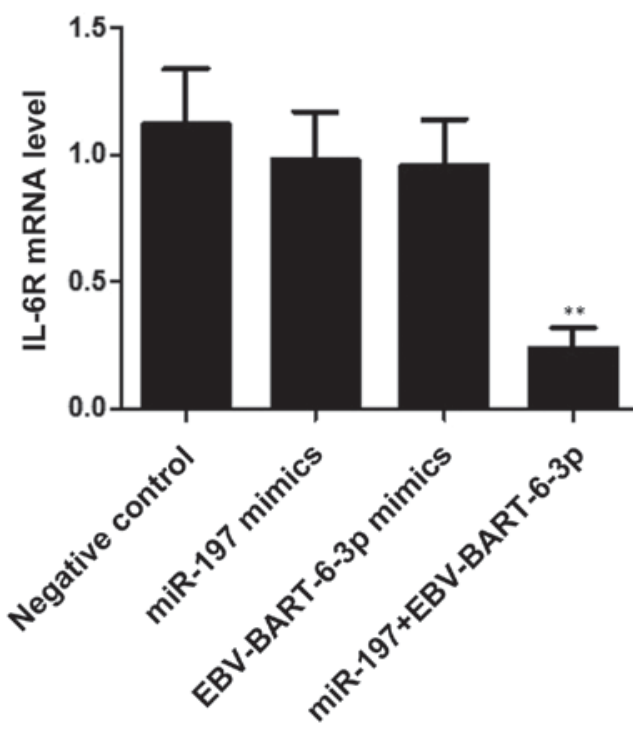

Figure 5. Effect of miR-197 and EBV-BART on IL-6R. (A) Protein levels of IL-6R decreased in cells treated with miR-197 or miR-BART6-3p mimics individually, compared with the negative control. The level of IL-6R in cells treated with miR-197 and miR-BART6-3p mimics together exhibited a marked decrease, compared with that in the negative control. (B) Expression level of IL-6R mRNA decreased marginally in cells treated with miR-197 mimics or miR-BART6-3p mimics individually, compared with the negative control, In the cells treated with miR-197 mimics and miR-BART6-3p mimics together, mRNA levels of IL-6R were markedly decreased, compared with the negative control. ${ }^{* *} \mathrm{P}<0.01$ vs. control group. miR, microRNA; EBV-BART-6-3p, Epstein-Barr virus-BamHI A region rightward transcript-6-3p; IL-6R, interleukin-6 receptor.

examined the mRNA and protein expression levels of IL-6R in ramos cells transfected with cellular miRNA mimics, EBV miRNA mimics, cellular miRNA and EBV miRNA, compared with the negative control. As shown in Fig. 5A, protein expression levels in ramos cells individually treated with miR-197 mimics or EBV-BART-6-3p mimics were mildly, but detectably downregulated, whereas the protein expression of IL-6R in the ramos cells treated with miR-197 and EBV-BART-6-3p together was markedly decreased, compared with the negative control. As shown in Fig. 5B, the mRNA expression in the ramos cells individually treated with miR-197 mimics or EBV-BART-6-3p mimics was marginally downregulated, whereas the mRNA expression of IL-6R in the ramos cells treated with miR-197 and EBV-BART-6-3p together was markedly lower, compared with that of the negative control cells. The above findings showed that the effect of cellular/EBV
miRNAs upon the target, IL-6R, was increased with the presence of cellular miRNA and EBV miRNA together, indicating the synergistic association between miR-BART6-3p and miR-197 on the expression of IL-6R.

\section{Discussion}

In the present study, cellular miR-197 was selected as the focus of investigation due to a previous report that it is differentially expressed and the observation that IL-6R is a virtual target of miR-197 and EBV-BART-6-3p (19). In the present study, IL-6R was confirmed as a target of cellular miR-197 using a luciferase assay, and the negative regulatory association between miRNA (miR-197 and EBV-BART-6-3p) and mRNA (IL-6R) was confirmed by the observation that IL-6R was downregulated in EBV-positive BL, whereas miR-197 was upregulated. Additionally, mimics of EBV-BART-6-3p and miR-197 were introduced into lymphoma cells, and it was found that EBV-BART-6-3p and miR-197 synergistically reduced the expression of IL-6R.

miR-197 has been found to be functionally involved in a number of human malignancies. It has been previously shown that dysregulation of miR-197 occurs in pancreatic cancer (21), tongue cancer (22), thyroid cancer (23) and lung cancer (24), which indicates that miR-197 has a general function in different types of tumor. In addition, previous studies have reported that the downregulation of miR-197 may lead to multidrug resistance in head and neck cancer cells (25) and colon cancer cells (26), suggesting that miR-197 is crucial in the regulation of several signaling pathways, and is important in the development of chemoresistance and tumor progression. miR-197 has been reported to be involved in the control of oncogenesis via targeting various genes. miR-197 has been reported to be one of the important miRNAs involved in uterine leiomyoma (27). Previous studies have shown that miR-197 is dysregulated in several tumor tissues and is associated with the proliferation and apoptosis of tumor cells. Du et al found that the levels of miR-197, a negative regulator of the expression of Fus1 in lung cancer is higher in non-small cell lung cancer cell lines and small cell lung cancer cell lines, compared with those in human bronchial epithelial cells (24). Hamada et al (21) found that the expression of miR-197 is significantly upregulated in invasive ductal adenocarcinoma, and the overexpression of miR-197 in pancreatic cancer cells targets p120 catenin, which contributes to the induction of epithelial-mesenchymal transition. In addition, anti-miR-197 inhibits the migration of hepatocellular carcinoma cells by targeting kaingai $1 /$ cluster of differentiation 82 (28). In the present study, it was found that the expression of miR-197 was markedly upregulated in EBV-positive BL samples, compared with EBV-negative controls. In addition, IL-6R was identified as a target of miR-197 and EBV-BART-6-3p, with putative binding sites in the 3'UTR of IL-6R. Furthermore, using a luciferase assay, IL-6R was confirmed as a shared target of miR-197 and EBV-BART-6-3p, with the binding site of miR-197 located at 2,838-2,845 bp within the 3'UTR of IL-6R.

ILs are responsible for anti-inflammatory and inflammatory responses of the immune system, and control the migration, differentiation and growth of cells. Cytokines may be critical in the carcinogenic process as they act as 
key regulators in the immune response. It is essential to maintain the balance between anti-inflammatory and inflammatory actions in order to protect tissues from underlying damage and to properly control the immune response (29). The IL-6/IL-6R signaling pathway is critical for the regulation of acute-phase responses, immune reactions and hematopoiesis (30). In addition, IL-6/IL-6R has been found to enhance the induction of lymphokine-activated killer cells (31), promote the differentiation and growth of $\mathrm{T}$ cells (32), and induce IgG secretion and B-cell differentiation (33). IL-6R is a heterodimeric receptor, composed of the $\alpha$ subunit ( $\mathrm{p} 80$ ), which is specific for IL- 6 binding, and the $\beta$ chain (gp130), which is the common transducer of different cytokines belonging to the IL-6 family (34). The IL-6 receptor regulates key cellular processes, including cell proliferation, cell survival, and the response to host pathogens following the binding of the dimerized receptor with its specific cytokine (interferon- $\alpha$, IL-12 or IL-27) $(34,35)$. A previous study showed that IL-6R is downregulated in EBV-positive nasopharyngeal carcinoma primary tumors and determines the lack of activation of the Janus kinase 2/signal transducer and activator of transcription pathway following IL-27 release, resulting in impairment of natural killer cell function and immune surveillance (36). It has also been shown that the downregulation of IL-6R in EBV-positive BL primary tumors and derived cell lines may be due to EBV-BART-6-3p, providing additional functional effects of EBV in evading the immune response and contributing to tumorigenesis (19). In the present study, it was found that the mRNA expression of IL-6R in EBV-positive samples was lower, compared with that in EBV-negative BL samples, and the protein expression of IL-6R was notably decreased in the EBV-positive samples, compared with the EBV-negative samples. In addition, the protein expression levels in ramos cells individually treated with miR-197 mimics or EBV-BART-6-3p mimics were mildly but detectably downregulated, whereas the protein expression levels of IL-6R in the ramos cells treated with miR-197 and EBV-BART-6-3p were markedly decreased, compared with the negative controls, indicating the synergistic association between miR-BART6-3p and miR-197 on the expression of IL-6R.

Taken together, the results of the present study demonstrated that high expression levels of miR-197 suppressed the expression of miR-197 synergistically with EBV-BART-6-3p, which compromised the immune defense system and contributed to the tumorigenesis of BL. miR-197 and EBV-BART-6-3p may offer potential as novel therapeutic targets in the treatment of BL.

\section{References}

1. Burkitt D: A sarcoma involving the jaws in African children. $\mathrm{Br}$ J Surg 46: 218-223, 1958.

2. Magrath I: Epidemiology: Clues to the pathogenesis of Burkitt lymphoma. Br J Haematol 156: 744-756, 2012.

3. Epstein MA and Barr YM: Cultivation in vitro of human lymphoblasts from Burkitt's malignant lymphoma. Lancet 1: 252-253, 1964

4. Dave SS, Fu K, Wright GW, Lam LT, Kluin P, Boerma EJ, Greiner TC, Weisenburger DD, Rosenwald A, Ott G, et al: Molecular diagnosis of Burkitt's lymphoma. N Engl J Med 354: 2431-2442, 2006.
5. Qiu J, Cosmopoulos K, Pegtel M, Hopmans E, Murray P, Middeldorp J, Shapiro M and Thorley-Lawson DA: A novel persistence associated EBV miRNA expression profile is disrupted in neoplasia. PLoS Pathog 7: e1002193, 2011.

6. Dalla-Favera R, Bregni M, Erikson J, Patterson D, Gallo RC and Croce CM: Human c-myc onc gene is located on the region of chromosome 8 that is translocated in Burkitt lymphoma cells. Proc Natl Acad Sci USA 79: 7824-7827, 1982.

7. Zimonjic DB, Keck-Waggoner C and Popescu NC: Novel genomic imbalances and chromosome translocations involving c-myc gene in Burkitt's lymphoma. Leukemia 15: 1582-1588, 2001.

8. Piccaluga PP, De Falco G, Kustagi M, Gazzola A, Agostinelli C, Tripodo C, Leucci E, Onnis A, Astolfi A, Sapienza MR, et al: Gene expression analysis uncovers similarity and differences among Burkitt lymphoma subtypes. Blood 117: 3596-3608, 2011.

9. Lenze D, Leoncini L, Hummel M, Volinia S, Liu CG, Amato T, De Falco G, Githanga J, Horn H, Nyagol J, et al: The different epidemiologic subtypes of Burkitt lymphoma share a homogenous micro RNA profile distinct from diffuse large B-cell lymphoma. Leukemia 25: 1869-1876, 2011.

10. Bartel DP: MicroRNAs: Genomics, biogenesis, mechanism, and function. Cell 116: 281-297, 2004.

11. Cullen BR: Transcription and processing of human microRNA precursors. Mol Cell 16: 861-865, 2004.

12. Bartel DP: MicroRNAs: Target recognition and regulatory functions. Cell 136: 215-233, 2009.

13. Cai X, Schäfer A, Lu S, Bilello JP, Desrosiers RC, Edwards R, Raab-Traub N and Cullen BR: Epstein-Barr virus microRNAs are evolutionarily conserved and differentially expressed. PLoS Pathog 2: e23, 2006

14. Skalsky RL, Corcoran DL, Gottwein E, Frank CL, Kang D, Hafner M, Nusbaum JD, Feederle R, Delecluse HJ, Luftig MA, et al: The viral and cellular microRNA targetome in lymphoblastoid cell lines. PLoS Pathog 8: e1002484, 2012.

15. Feederle R, Linnstaedt SD, Bannert H, Lips H, Bencun M, Cullen BR and Delecluse HJ: A viral microRNA cluster strongly potentiates the transforming properties of a human herpesvirus. PLoS Pathog 7: e1001294, 2011.

16. Seto E, Moosmann A, Grömminger S, Walz N, Grundhoff A and Hammerschmidt W: Micro RNAs of Epstein-Barr virus promote cell cycle progression and prevent apoptosis of primary human B cells. PLoS Pathog 6: e1001063, 2010.

17. Vereide DT, Seto E, Chiu YF, Hayes M, Tagawa T, Grundhoff A, Hammerschmidt W and Sugden B: Epstein-Barr virus maintains lymphomas via its miRNAs. Oncogene 33: 1258-1264, 2014.

18. Kanda T, Miyata M, Kano M, Kondo S, Yoshizaki T and Iizasa H: Clustered microRNAs of the Epstein-Barr virus cooperatively downregulate an epithelial cell-specific metastasis suppressor. J Virol 89: 2684-2697, 2015.

19. Ambrosio MR, Navari M, Di Lisio L, Leon EA, Onnis A, Gazaneo S, Mundo L, Ulivieri C, Gomez G, Lazzi S, et al: The Epstein Barr-encoded BART-6-3p microRNA affects regulation of cell growth and immuno response in Burkitt lymphoma. Infect Agent Cancer 9: 12, 2014.

20. Livak KJ and Schmittgen TD: Analysis of relative gene expression data using real-time quantitative PCR and the 2(-Delta Delta C(T)) Method. Methods 25: 402-408, 2001.

21. Hamada S, Satoh K, Miura S, Hirota M, Kanno A, Masamune A, Kikuta K, Kume K, Unno J, Egawa S, et al: miR-197 induces epithelial-mesenchymal transition in pancreatic cancer cells by targeting p120 catenin. J Cell Physiol 228: 1255-1263, 2013.

22. Wong TS, Liu XB, Wong BY, Ng RW, Yuen AP and Wei WI: Mature miR-184 as potential oncogenic microRNA of squamous cell carcinoma of tongue. Clin Cancer Res 14: 2588-2592, 2008.

23. Keutgen XM, Filicori F, Crowley MJ, Wang Y, Scognamiglio T, Hoda R, Buitrago D, Cooper D, Zeiger MA, Zarnegar R, et al: A panel of four miRNAs accurately differentiates malignant from benign indeterminate thyroid lesions on fine needle aspiration. Clin Cancer Res 18: 2032-2038, 2012.

24. Du L, Schvageman JJ, Subauste MC, Saber B, Hammond SM, Prudkin L, Wistuba II, Ji L, Roth JA, Minna JD and Pertsemlidis A: miR-93, miR-98, and miR-197 regulate expression of tumor suppressor gene FUS1. Mol Cancer Res 7: 1234-1243, 2009.

25. Dai Y, Xie CH, Neis JP, Fan CY, Vural E and Spring PM: MicroRNA expression profiles of head and neck squamous cell carcinoma with docetaxel-induced multidrug resistance. Head Neck 33: 786-791, 2011. 
26. Zhou J, Zhou Y, Yin B, Hao W, Zhao L, Ju W and Bai C: 5-Fluorouracil and oxaliplatin modify the expression profiles of microRNAs in human colon cancer cells in vitro. Oncol Rep 23: 121-128, 2010.

27. Wang T, Zhang X, Obijuru L, Laser J, Aris V, Lee P, Mittal K, Soteropoulos P and Wei JJ: A micro-RNA signature associated with race, tumor size, and target gene activity in human uterine leiomyomas. Genes Chromosomes Cancer 46: 336-347, 2007.

28. Dai W, Wang C, Wang F, Wang Y, Shen M, Chen K, Cheng P, Zhang Y, Yang J, Zhu R, et al: Anti-miR-197 inhibits migration in HCC cells by targeting KAI 1/CD82. Biochem Biophys Res Commun 446: 541-548, 2014.

29. DeNardo DG and Coussens LM: Inflammation and breast cancer. Balancing immune response: Crosstalk between adaptive and innate immune cells during breast cancer progression. Breast Cancer Res 9: 212, 2007.

30. Kishimoto T: The biology of interleukin-6. Blood 74: 1-10, 1989.

31. Lu LS: Experimental study on IL-6 activity against tumor: I Regulation of IL-2-induced LAK cells. Zhonghua Zhong Liu Za Zhi 14: 250-252, 1992 (In Chinese).
32. Mizutani H, May LT, Sehgal PB and Kupper TS: Synergistic interactions of IL-1 and IL-6 in T cell activation. Mitogen but not antigen receptor-induced proliferation of a cloned Thelper cell line is enhanced by exogenous IL-6. J Immunol 143: 896-901, 1989.

33. Muraguchi A, Kishimoto T, Miki Y, Kuritani T, Kaieda T, Yoshizaki $\mathrm{K}$ and Yamamura Y: T cell-replacing factor- (TRF) induced IgG secretion in a human B blastoid cell line and demonstration of acceptors for TRF. J Immunol 127: 412-416, 1981

34. Kishimoto T: Interleukin-6: From basic science to medicine-40 years in immunology. Annu Rev Immunol 23: 1-21, 2005.

35. Peters M, Jacobs S, Ehlers M, Vollmer P, Müllberg J, Wolf E, Brem G, Meyer zum Büschenfelde KH and Rose-John S: The function of the soluble interleukin 6 (IL-6) receptor in vivo: Sensitization of human soluble IL-6 receptor transgenic mice towards IL-6 and prolongation of the plasma half-life of IL-6. J Exp Med 183: 1399-1406, 1996.

36. Lin YS, Lin LC, Huang MH, Huang AJ and Huang YT: Down-regulation of gp130 in nasopharyngeal carcinoma. Am J Rhinol Allergy 23: 28-32, 2009. 